\title{
Allogeneic blood-based therapies: hype or hope?
}

Eye (2017) 31, 509-510; doi:10.1038/eye.2016.268; published online 2 December 2016

The use of allogeneic blood therapies has opened new avenues in several medical fields including ophthalmology. ${ }^{1}$ Blood-based platelet and plasma products mimic natural tears because of their content in growth factors, cytokines, vitamins and nutrients, which are essential for maintaining the homeostasis of the normal ocular surface tissues. ${ }^{1}$ Although autologous eye drops are commonly used in the management of ocular disorders, not all patients are candidates as donors due to systemic inflammatory diseases, age, and other types of disorders or comorbidities. In these cases, an allogeneic blood-based product may represent an alternative to manage several ocular surface injuries, such as severe dry eye and persistent corneal epithelial defects. ${ }^{2,3}$ The use of allogeneic therapies has major concerns, including hypersensitivity reactions in the recipient, the risk of blood-borne pathogen infection, and the requirement of tests for transfusiontransmissible infections and virus screening in the eye drops. Recently, solvent/detergent virally inactivated blood-based eye drops and the photochemical treatment of the allogeneic formulations have been proposed for virus inactivation. ${ }^{4}$

It is well known that the eye is an immuneprivileged organ. Assuming that ABO and HLA antigens are present on conjunctival and corneal surfaces, blood-based products could trigger an inflammatory response by complement activation. Our group proposed an inactivation protocol of immune components in the donor's eye drops, which preserves most of the biologically active agents while reducing drastically complement activity and immunoglobulin content. ${ }^{5}$

Several hurdles need to be overcome in order to make further progress in the use of allogeneic eye drops. One particular challenge relies on the manufacturing processes. Standardized protocols to obtain human blood-derived products should be regulated by individual laws of each country and the devices designed for obtaining these products should be strictly controlled. Storage conditions should also be optimized for future progress of the approach.

Allogeneic blood-based therapies are already in their infancy. The potential benefits of treating diseases by using allogeneic products are enormous, but, unfortunately, this technology still faces several significant challenges, including safety, a harmonization of legislation in this matter, and more rigorous policies of manufacturing and standardization of these products.

\section{Conflict of interest}

The authors declare that EA is the Scientific Director and MDIF, GO and FM are scientists at BTI Biotechnology Institute, a dental implant company that investigates in the fields of oral implantology and PRGF-Endoret technology.

\section{References}

1 Anitua E, Muruzabal F, Tayebba A, Riestra A, Perez VL, Merayo-Lloves J et al. Autologous serum and plasma rich in growth factors in ophthalmology: preclinical and clinical studies. Acta Ophthalmol 2015; 93(8): e605-e614.

2 Chiang CC, Chen WL, Lin JM, Tsai YY. Allogeneic serum eye drops for the treatment of persistent corneal epithelial defect. Eye (Lond) 2009; 23(2): 290-293.

3 Na KS, Kim MS. Allogeneic serum eye drops for the treatment of dry eye patients with chronic graftversus-host disease. J Ocul Pharmacol Ther 2012; 28(5): 479-483.

4 Prowse CV. Component pathogen inactivation: a critical review. Vox Sang 2013; 104(3): 183-199.

5 Anitua E, Muruzabal F, De la Fuente M, Merayo-Lloves J, Orive G. Effects of heattreatment on plasma rich in growth factors-derived autologous eye drop. Exp Eye Res 2014; 119: $27-34$. 
E Anitua ${ }^{1,2}$, M de la Fuente ${ }^{2}$, J Merayo-Lloves ${ }^{3}$, F Muruzabal $^{2}$ and G Orive $1,2,4,5$

${ }^{1}$ Eduardo Anitua Foundation for Biomedical Research, Vitoria, Spain

${ }^{2}$ Research and Development, BTI - Biotechnology Institute, Vitoria, Spain

${ }^{3}$ Instituto Universitario Fernández-Vega, Universidad de Oviedo, Oviedo, Spain

${ }^{4}$ Laboratory of Pharmacy and Pharmaceutical Technology, Faculty of Pharmacy, University of the Basque Country, Vitoria, Spain
${ }^{5}$ Networking Biomedical Research Center on Bioengineering, Biomaterials and Nanomedicine, CIBER-BBN, SLFPB-EHU, Vitoria, Spain

Correspondence: G Orive, Research and Development, BTI - Biotechnology Institute, Jacinto Quincoces, 39, Vitoria 01007, Alava, Spain

Tel: +34 945 160653;

Fax: +34945 154187 .

E-mail: gorka.orive@ehu.es 